\title{
Sistema computacional para el control y monitoreo de la cocina de inducción por internet
}

\section{Computer system for controlling and monitoring of induction cooker through Internet}

\author{
Manuel Alexander Ponce Tubay ${ }^{1, *}$, Sonia Monserrate Párraga Muñoz ${ }^{1, \dagger}$, y Jhonny Andrés Ochoa Parrales ${ }^{1, \ddagger}$ \\ ${ }^{1}$ Universidad San Gregorio de Portoviejo. \\ \{maponce,smparraga,e.jaochoa\}@sangregorio.edu.ec
}

Fecha de recepción: 15 de agosto de 2017 — Fecha de aceptación: 15 de septiembre de 2017

\begin{abstract}
Cómo citar: Ponce Tubay, M. A., Párraga Muñoz, S. M., \& Ochoa Parrales, J. A. (2018). Sistema computacional para el control y monitoreo de la cocina de inducción por internet. Journal of Science and Research: Revista Ciencia e Investigación, 3(CITT2017), 61-64. https://doi.org/10.26910/issn.2528-8083vol3issCITT2017.2018pp65-68
\end{abstract}

\begin{abstract}
Resumen-En el Ecuador, el gobierno estableció reformas a la matriz energética, entre las cuales se hizo referencia a la implementación de las cocinas de inducción, un programa denominado Programa de Eficiencia para cocción por inducción y calentamiento de agua con electricidad, en el cual se incentiva a las personas a la utilización de las mismas. El presente proyecto se realizó en la ciudad de Portoviejo y se enfoca en un componente de innovación que permite controlar la cocina de inducción desde cualquier parte mediante internet. Se identificó que actualmente no existe un sistema que permita realizar dichas operaciones que permiten realizar de forma eficaz las labores de cocción, a su vez, con las debidas medidas de control puede evitar accidentes dentro de la cocina, en específico con menores de edad o personas que no saben utilizar la cocina de inducción. El conjunto de actividades planteadas permitieron dar cumplimiento a los objetivos de este proyecto, finalizando con la realización del sistema computacional para el control y monitoreo de la cocina de inducción por internet.
\end{abstract}

Palabras Clave-Campo electromagnético, Inducción, Inductores, Dispositivos móviles.

\begin{abstract}
In Ecuador, the government established reforms to the energy matrix, among which reference was made to the implementation of induction cookers, a program called Efficiency Program for induction cooking and water heating with electricity, which encourages people to use them. The present project was carried out in the city of Portoviejo and focuses on an innovation component that allows controlling the induction cooker from anywhere using the internet. It was identified that at the moment there is no system that allows to carry out such operations that allow performing the cooking effectively, in turn, with the appropriate control measures can avoid accidents within the kitchen, specifically with minors or people Who do not know how to use them induction cooker. The set of activities allowed to fulfill the objectives of this project, ending with the realization of the computer system for the control and monitoring of the induction cooker by the internet.
\end{abstract}

Keywords-Electromagnetic field, Induction, Inductors, Mobile devices.

\section{INTRODUCCIÓN}

$\mathbf{E}$ n Ecuador, estudiantes de la ESPE (Escuela Superior Politécnica del Ejercito) inspirados por el inminente cambio de la matriz productiva y energética fomentado por el Plan Nacional del Buen Vivir, realizaron una investigación denominada "Diseño e implementación de calefón de inducción electromagnética" (Rivera Coque, 2013), este fue el primer estudio en el cual se diseña y fabrica el primer modelo ecuatoriano de cocina de inducción en el Ecuador, posterior a eso en Guayaquil presentan la investigación "Cambio en la matriz energética y su efecto en la producción nacional: caso cocina de inducción" (LUCAS CARBO, 2015) que incentiva a sus habitantes a hacer uso de las cocinas de inducción para así reducir el consumo de Gas licuado de petróleo, a partir de este momento se han desarrollado diversas investigaciones referentes a las cocinas de inducción, preparando la mejor

\footnotetext{
* Master en Seguridad Informática Aplicada

${ }^{\dagger}$ Master en Seguridad Informática Aplicada

¥Ingeniero en Ciencias de la Computación
}

solución para el inminente cambio. Uno de ellos fue "Estudio de ensayos de eficiencia energética, concavidad, convexidad y rugosidad en menaje para cocinas de inducción" (Orozco, 2014). Realizado en Cuenca-Ecuador en el 2014. En el cual se estudian las propiedades ferromagnéticas de la base de la cocina y cambian ciertos tipos de materiales mejorando así la eficiencia energética, concavidad, convexidad y rugosidad de la cocina de inducción. Otro Estudio fue el realizado en la Universidad Politécnica Salesiana "Análisis del rendimiento térmico de una cocina de inducción de 3500W de potencia" (Amay Salinas, 2015) por Amay Salinas, Pedro Luis Riofrío Ordóñez, César Augusto en abril del 2015 en el cual lograron disminuir el consumo de energía aumentando la potencia de la cocina con la integración de un dispositivo regulador. Conociendo todo el antecedente proveniente de la cocina de inducción, nace la investigación en la cual se busca convertir la manera en la que se cocinan los alimentos, actualmente disminuyendo el tiempo necesario para esta, con el fin de alimentar la investigación se establece un componente importante de innovación que permite monitorear y controlar la cocina de 
inducción de manera remota facilitando las tareas de cocción a las personas que no cuentan con el tiempo necesario para realizarlo desde su hogar.

\section{Problematización}

La cocción de los alimentos ha sido, es, y será una de las principales necesidades del ser humano, pero en la sociedad actual cada vez se hace más difícil llevarlo a cabo, sobre todo por la inclusión de la mujer en el campo laboral las personas ya no disponen del tiempo suficiente para poder llegar a sus hogares, preparar los ingredientes, cocinarlos y disfrutar su comida, es uno de los principales problemas de la globalización, siendo esta una problemática vigente, porque no existen los estudios o investigaciones de tecnología que permitan al usuario operar la cocción de alimentos de forma remota. En nuestro país no han sido concretadas dichas investigaciones. Los artefactos o herramientas utilizados para la cocción de alimentos pueden provocar desmanes o eventos que atentan contra la salud del usuario, esto pasa por la poca seguridad que este tipo de electrodomésticos brindan al momento de su uso; esto genera un riesgo que involucra a las familias por el acercamiento de los niños a la cocina, para realizar el calentamiento debido de los alimentos. En ocasiones el padre no puede monitorear o realizar estas actividades, siendo riesgoso para el menor usar esta tecnología. La propiedad intelectual del fabricante de las cocinas de inducción ha ocasionado problemas en la creación de una tecnología que administre las mismas o un dispositivo que pueda controlarlas, dificultando por mucho tiempo la generación de nuevas aplicaciones en torno a esto. El acceso a internet en hogares ecuatorianos en tiempos pasado, era menos concurrentes que en la actualidad, donde las mayorías de personas cuentan con este servicio lo que facilita su adaptabilidad a los electrodomésticos. La creación de dispositivos tecnológicos que faciliten diferentes actividades que cotidianamente realizan las personas, en la actualidad es relevante, un problema latente es que no todas estas tecnologías son explotadas y a la vez con utilidades específicas, una referencia es el monitoreo visual en la web en tiempo real, que debe ser aprovechado por varias instancias, así como al momento de la cocción de alimentos en una cocina de inducción.

\section{JUSTIFICACIÓN}

La presente investigación se enfoca en la creación de un dispositivo que integrado a la cocina de inducción nos permita controlar y monitorear la misma, añadiendo funciones de fácil y rápido acceso a la configuración, todo esto sin la necesidad de estar presente en el momento de la cocción, permitiendo al usuario desde su trabajo o en cualquier lugar que se encuentre encenderla y administrarla desde su celular o cualquier dispositivo con acceso a internet.

La herramienta desarrollada, permitirá evitar riesgos y accidentes que comúnmente ocurren por descuido o el uso incorrecto de los artefactos o herramientas utilizados para la cocción de alimentos ya que con la integración de esta herramienta permitirá realizar un monitoreo de forma constante para verificar el estado de lo que en ese momento se está cocinando, además de controles de seguridad periódicos para evitar cualquier imprevisto, con esto se busca que las familias puedan llegar al hogar directamente a disfrutar de los alimentos.

La presente investigación plantea realizar el diseño de un componente tecnológico sin alterar la propiedad intelectual de la cocina de inducción, de esta forma obteniendo el consentimiento de los mismos para el estudio y desarrollo de nuevas tecnologías.

El número de hogares con acceso a internet ha incrementado gracias a las accesibilidades y beneficios que brindan este tipo de red inalámbricas, por ello esta investigación se ve beneficiada por la factibilidad que esto genera a la implementación de la herramienta en distintos hogares.

Con la ayuda de tecnologías de cámaras ip, se realiza la implementación del control y vigilancia en los procesos de cocción de alimentos, para de esta forma tener una imagen del entorno claro y ágil al momento de obtener la información, implementando así un módulo de monitoreo por video en tiempo real

\section{DESARROLLO}

\section{Tecnología de Inducción}

Para entender cómo funciona una cocina de inducción es necesario recordar brevemente el fenómeno de la inducción magnética. Los experimentos del Físico y Químico inglés Michael Faraday (1791-1867) en Inglaterra en 1831 y los efectuados de forma independiente por Joseph Henry en Estados Unidos, ese mismo año, mostraron que es posible inducir una fuerza electromotriz en un circuito, utilizando un campo magnético variable. Fue por los resultados obtenidos en esa investigación, los que sirvieron como pilar para definir una ley básica y de mucha importancia para el electromagnetismo conocida como la Ley de la Inducción de Faraday. Una corriente puede ser inducida en diferentes procesos que involucran un cambio en el flujo magnético (Franco, 2013).

En conclusión si una corriente eléctrica de frecuencia variable genera un campo magnético, dicho campo magnético variable induce una corriente eléctrica en un circuito cerrado; ésta última a su vez, se relaciona con un campo eléctrico no conservativo. En esencia, la cocina de inducción es un tipo de cocina vitro cerámica, cuyo elemento principal, ubicado debajo de la zona de cocción, es una bobina plana de cobre y con forma de espiral denominadas inductores, por la que se hace pasar una corriente eléctrica I de frecuencia variable $(20-100 \mathrm{kHz})$, la misma que genera una densidad de flujo magnético alterno, con la misma frecuencia con la que varía la corriente en la bobina. Campo Electromagnético.

El electromagnetismo es conocido por estudiar y determinar los fenómenos eléctricos y magnéticos en una sola teoría, cuyos fundamentos fueron presentados por Michael Faraday y formulados por primera vez de modo completo por James Clerk Maxwell. La formulación consiste en cuatro ecuaciones diferenciales vectoriales que relacionan el campo eléctrico, el campo magnético y sus respectivas fuentes materiales (corriente eléctrica, polarización eléctrica y polarización magnética), conocidas como ecuaciones de Maxwell. El electromagnetismo es una teoría de campos; es decir, las explicaciones 
y predicciones que provee se basan en magnitudes físicas vectoriales o tensoriales, dependientes de la posición en el espacio y del tiempo. El electromagnetismo se encarga de describir los fenómenos físicos en los cuales afectan cargas eléctricas en estado de reposo y movimiento (Serway, 2005).

En el caso propio de la cocina de inducción debido al instrumento que se usa para la cocción de alimentos (olla, cacerola, cazuela etc...) este se asienta en la zona de cocción que está muy próxima a la bobina espiral o inductor, el campo magnético, producido por esta última, será aproximadamente el mismo en toda esa región; en la siguiente imagen se trata de reflejar esta expresión:

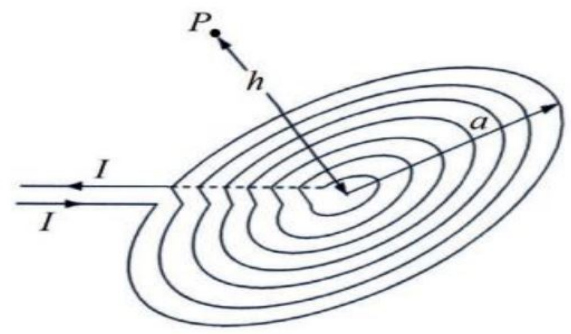

Figura 1. Propagación de campo electromagnético en la cocina de inducción.

Fuente: Elaboración Propia.

$$
\boldsymbol{B}(P)=\sum_{i=1}^{N} \frac{\mu_{0} I(a i)^{2}}{2 N^{2}\left[h^{2}+\left(\frac{a i}{N}\right)^{2}\right]^{3 / 2}} \boldsymbol{\mu}_{z}
$$

Figura 2. Propagación de campo electromagnético en la cocina de inducción.

Fuente: Elaboración Propia.

Dónde a es el radio de la bobina plana de cobre y con forma espiral, h es la altura por encima de la bobina a la cual se está determinando la densidad de flujo magnético. $\mathrm{N}$ es el número de espiras o de vueltas que tiene la precitada bobina, asumiendo que se encuentran uniformemente distribuidas y que cada espira o vuelta es una espiral circular perfecta, i es el elemento espira circular perfecta, que va desde 1 hasta (Franco, 2013).

\section{LEY DE INDUCCIÓN DE FARADAY}

Alrededor de los años 30, Michael Faraday descubrió que cuando un conductor que forma un circuito cerrado se mueve por algún medio mecánico, en un campo magnético, un voltaje se produce de alguna manera y este produce una circulación de corriente. Asociado al descubrimiento de Faraday, se supo también el hecho de que el magnetismo se puede generar por una corriente eléctrica que pasa a través de una bobina y que los polos iguales de un imán se repelen, en tanto los contrarios se atraen. Estos fenómenos, asociados a ciertas reglas y leyes eléctricas, constituyen la base para el estudio de los generadores y motores eléctricos.

En si esta ley se puede definir como "El voltaje inducido en una espiral o bobina de un conductor, es proporcional al índice de cambio de las líneas de fuerza que pasan a través de la bobina" (Harper, 1987). La ley de inducción de Faraday, aporta los fundamentos físicos necesarios para la conceptualización de la fuerza electromotriz inducida en las bobinas de las máquinas eléctricas en general. Entre estas máquinas se pueden mencionar las siguientes: A) Motores y generadores de corriente continua, B) Transformadores, C) Motores asincrónicos trifásicos y monofásicos y D) Generadores y motores sincrónicos. La ley de Faraday tiene la forma general:

$$
e=-\frac{d \varnothing}{d t}
$$

Donde e es la fuerza electromotriz inducida y es el flujo del campo magnético, definido como la integral del producto entre la intensidad de campo magnético y la sección normal al campo.

Si se tiene una bobina de sección transversal constante para todas las $\mathrm{N}$ espiras, y el flujo de campo magnético abraza a todas por igual, la ecuación adopta la forma siguiente:

$$
e=-N \frac{d \varnothing}{d t}
$$

La razón de la introducción de esta ley estriba en que las máquinas eléctricas, de corriente continua o de corriente alterna, están constituidas por espiras que al ser abrazadas por campos magnéticos variables en el tiempo (proveniente de otra fuente $u$ originados por su propia corriente), generan fuerzas electromotrices (f.e.m.) inducidas que a su vez dan origen a las corrientes que circulan por los circuitos eléctricos de los generadores y secundarios de los transformadores, o fuerzas contra electromotrices auto inducidas que se oponen a la circulación de corriente como en los motores y primarios de los transformadores. La explicación de esta se fundamenta en la ley de la inducción de Faraday (Sánchez, 2001).

7.2.1.2 Elementos que conforman un sistema de calentamiento por inducción Los elementos básicos que componen o comprenden un sistema de calentamiento por inducción son:

Sistema de alimentación eléctrica

Se encarga de suministrar la energía necesaria para todo el sistema, dependiendo de la potencia y el tipo de conversor, en la mayoría de los casos necesita de una fuente de alimentación de $220 \mathrm{v}$.

Fase de rectificación

Esta fase es la encargada de convertir la corriente alterna (AC) de la fuente de energía eléctrica en corriente continua (DC) mediante un vector de diodos.

Inversor de alta frecuencia

Esta fase convierte las corrientes a una frecuencia de red eléctrica con el objetivo de que esta sea la encargada de 
alimentar los inductores de calentamiento o la bobina de trabajo. Bobinas de Trabajo Las bobinas de trabajo son las encargadas de la generación de los campos magnéticos en las cercanías del material a calentar.

Sistema de refrigeración

El inductor y el resto de componentes de la cocina de inducción acumulan gran cantidad de potencias y necesitan disiparlas, el sistema de refrigeración es el encargado de realizar este proceso mediante la utilización de algún elemento refrigerante que por lo general es suficiente con el uso de ventiladores.

Sistema de Control

Es el encargado de la emisión de señales digitales destinadas a la placa de control la cual se encarga de determinar y decretar las funciones del sistema de inducción: encendido, potencia, temporizador etc...

Elemento de calentamiento

Por lo general el elemento a calentar debe ser de algún tipo de material ferromagnético, ya que este tipo de material se calienta más fácilmente debido al calentamiento adicional por el efecto de histéresis. En general todas las fases y componentes anteriormente descritos son los necesarios para implementar un sistema de calentamiento por inducción (Collaguazo, 2009).

\section{CONCLUSIONES}

El Sistema computacional para el control y monitoreo de la cocina de inducción por internet que se implementó, mostró la utilidad y la importancia de componentes de innovación en esta herramienta tan primordial como es la cocina.

La implementación de este tipo de sistemas en conjunto con herramientas tecnológicas puede traer grandes beneficios en muchas áreas, no sólo en la cual el proyecto ha sido enfocado. Se pudo evidenciar de esta manera que varias tecnologías que conocemos habitualmente comunes, permiten realizar tareas mucho más avanzadas y útiles como para ser implementadas en este tipo de investigación.

El impacto obtenido con la implementación de esta investigación fue de mucho beneficio para los usuarios finales de la cocina de inducción, pues resuelve la problemática planteada en este mismo.

\section{REFERENCIAS BIBLIOGRÁFICAS}

Adell, J. and Bellver, C. (1995). La internet como teleraña: el world-wide web. Métodos de información, 2(3):25-32.

Aguirre Pineda, A. X. (2016). Elaboración de una guía metodológica para la investigación de proyectos contables en la ciudad de machala.

Amay Salinas, P. L. and Riofrío Ordoñez, C. A. (2015). Análisis del rendimiento térmico de una cocina de inducción de $3500 \mathrm{w}$ de potencia. B.S. thesis.

Barreno Masabanda, F. M. et al. (2013). Diseño de prototipo doméstico de video vigilancia con cámaras ip por internet. B.S. thesis, Quito, 2013.

Cisneros, J. T. C., Trejo, C. A., Bermeo, M. F. C., et al. (2014). El impacto que tendría el cambio de cocinas a gas por inducción en la sociedad ecuatoriana. Observatorio de la Economía Latinoamericana, (202).

Collaguazo, C., Antonio, E., Saráuz, T., Gonzalo, D., and Sotomayor, N. (2009). Diseño y construcción de un prototipo de una cocina de inducción electromagnética. $\mathrm{PhD}$ thesis.

Crespo, G. O. (2013). La sustitución de cocinas: una ruta para rebajar el subsidio. Revista Gestión.

Franco, T. and Alberto, G. (2013). Cocina de inducción versus cocina a gas (glp).

Harper, G. E. (1987). El ABC de las máquinas eléctricas, volume 2. Editorial Limusa.

LUCAS CARBO, D. M. (2015). Cambio en la matriz energética y su efecto en la producción nacional: caso cocina de inducción del cantón Guayaquil. $\mathrm{PhD}$ thesis, Universidad de Guayaquil Facultad de Ciencias Económicas.

Mera Cedeño, J. V. (2017). Sistema computacional para el control y monitoreo de la cocina de inducción por internet.

Morales Torres, P. T. et al. (2012). Sistema prototipo de telemonitoreo remoto de signos vitales como la frecuencia cardíaca, usando tecnologías inalámbricas de comunicación disponibles en los teléfonos celulares. B.S. thesis, Quito, 2012.

Orozco, M., Martínez, J., Riofrío, A., Vaca, D., and Carrión, D. (2014). Estudio de ensayos de eficiencia energética, concavidad, convexidad y rugosidad en menaje para cocinas de inducción. In Memorias del Congreso latinoamericano de ingeniería mecánica Colim, volume 8, pages 255-261.

Rivera Coque, A. R. and Flores Gutiérrez, L. A. (2016). Diseño e implementación de un calefón a inducción electromagnética para proveer de agua caliente a viviendas unifamiliares. B.S. thesis, Universidad de las Fuerzas Armadas ESPE Extensión Latacunga. Carrera de Ingeniería en Electrónica e Instrumentación.

Ruiz, L. (1997). Versión avanzada de un sistema computacional aplicado a una investigación lexicológica. Estudios de Comunicación Social, Editorial Academia, La Habana, pages $85-113$.

Sánchez, D. N. (2001). La ley de inducción de faraday. una propuesta para la enseñanza media técnica. 